\title{
Electrochemical Characterisation of Nanoscale Liquid|Liquid Interfaces \\ Located at Focused Ion Beam-Milled Silicon Nitride Membranes
}

Masniza Sairi, ${ }^{[a, b]}$ Nigel Chen-Tan, ${ }^{[c]}$ Gregor Neusser, ${ }^{[d]}$ Christine Kranz, ${ }^{[\mathrm{d}]}$ and Damien W. M. Arrigan $^{[\mathrm{a}]}$ *

[a]M. Sairi and Prof. Dr. D. W. M. Arrigan

Nanochemistry Research Institute, Department of Chemistry,

Curtin University,

GPO Box U1987, Perth, Western Australia 6845, Australia

Email: d.arrigan@curtin.edu.au

[b]M. Sairi

Mechanisation and Automation Research Centre,

Malaysian Agricultural Research and Development Institute (MARDI),

P.O. Box 12301, 50774 Kuala Lumpur, Malaysia

[c]Dr. N. Chen-Tan

John de Laeter Centre, Department of Imaging and Applied Physics,

Curtin University,

GPO Box U1987, Perth, Western Australia 6845, Australia

[d]G. Neusser and Dr. C. Kranz

Institute of Analytical and Bioanalytical Chemistry,

University of Ulm,

Albert-Einstein-Allee 11, 89081 Ulm, Germany

Abstract: The electrochemical behaviour of single and arrayed nanoscale interfaces between
two immiscible electrolyte solutions (single and array nanolTIES) is presented. The interfaces 
were formed at nanopores fabricated via focused ion beam (FIB)-milling of silicon nitride ( $\mathrm{SiN}$ ) membranes using nanopores in the range of ca. 30 to $80 \mathrm{~nm}$ in radii and with pore-to-pore separation to pore radius ratios in the range of 16 to 32 . Electrochemistry was performed by the interfacial transfer of tetrapropylammonium (TPrA ${ }^{+}$) across single and array nanolTIES between water and 1,6-dichlorohexane. The ion-transfer limiting current at the single nanoITIES was in excellent agreement with the current predicted using an inlaid disc interface model. At nanoITIES arrays, experimental currents were lower than predicted for an array of inlaid interfaces, attributed to overlapped diffusion zones. As a result, FIB milling offers an attractive strategy to form nanoITIES for diverse investigations.

\section{Introduction}

Electrochemistry at the interface between two immiscible electrolyte solutions (ITIES) ${ }^{[1]}$ or at liquid | liquid interfaces, is of great importance in analytical chemistry because it allows the detection of species and processes in a label-free manner even if the oxidation or reduction reaction at a solid electrode is difficult or is masked by an interfering constituent ${ }^{[2]}$. As discussed in some recent reviews, electrochemical sensing based on ion-transfer across the ITIES has focused on the detection of biologically-relevant molecules such as proteins, peptides, amino acid and small molecules (drugs, neurotransmitters and food additives) ${ }^{[2-3]}$.

The miniaturisation of the ITIES to nanoscale dimensions ${ }^{[4]}$ offers analogous benefits to those of nanoelectrode arrays, namely enhanced mass transport flux, increased current densities, and enhanced sensitivity of the analytical response ${ }^{[5]}$. To date, two approaches have been extensively employed in the formation of nanoscale ITIES. The first approach is based on supporting the liquid | liquid interface at the tip of a nanopipette ${ }^{[6]}$ or a double-barrelled nanopipette [6c, 7], which allows the establishment of single or double nanointerfaces, respectively. The second approach is based on supporting the interface at the orifices of geometrically irregular or regular nanopore arrays in membranes ${ }^{[5 a, 5 c, 8]}$. Track-etched polyester 
${ }^{[8 a, 8 b]}, \gamma$-alumina ultrafiltration ${ }^{[8 c]}$ and silicon nitride $(\mathrm{SiN})$ membranes ${ }^{[5 a, 5 c]}$ have been reported as substrates for irregular and regular nanoITIES arrays.

The fabrication of solid-state pores with nanometre dimensions is challenging and several approaches have been examined, such as focused ion beam (FIB) milling ${ }^{[9]}$, direct drilling using the high-energy focused electron beams of transmission electron microscopy (TEM) [10], electron beam lithography $(\mathrm{EBL})$ and chemical etching ${ }^{[5 a, 11]}$, reactive gas- or water vapourassisted charge beam etching ${ }^{[12]}$, and track etching by high-energy heavy metal ions combined with chemical etching ${ }^{[8 a, 8 b]}$. FIB milling provides an alternative to EBL since it is a direct-write method, and thus facilitates the fast prototyping of nanopore membranes ${ }^{[13]}$. EBL also involves more complex and time-consuming procedures ${ }^{[13]}$. SiN and silicon dioxide are the most widely used materials for nanopore structures generated by $\mathrm{FIB}^{[14]}$, since these materials are widelyused in microelectronics fabrication ${ }^{[15]}$.

Inorganic, solid-state nanopore membranes prepared by FIB milling and TEM electron beam drilling have been reported for DNA sequencing ${ }^{[15-16]}$, building on biomolecule translocation through $\alpha$-haemolysin protein nanopores ${ }^{[17]}$. These translocation studies ${ }^{[17]}$ have triggered interest in the development of solid-state nanopores in insulating membranes. The significant advantages offered by solid-state nanopore membranes include high mechanical, chemical and electrical stability, rigidity, modifiable surface properties, geometry control, and the prospect for integration into devices and arrays ${ }^{[10 a, 12 b, 14-15,18]}$. To-date, the performance of such FIB-milled nanopore membranes as platforms for nanoITIES and nanoelectrochemical sensing has not been examined, and, accordingly, that is the focus of this study.

Here, the preparation of single and array nanopores via FIB milling and the characterisation of the ITIES localised within these FIB-generated nanopores is presented. The behaviour of the nanolTIES was characterised by cyclic voltammetry (CV) via the transfer of tetrapropylammonium $\left(\mathrm{TPrA}^{+}\right)$across the water|1,6-dichlorohexane (DCH) interface. The voltammetric responses relative to the number of pores in the array, and the ratio between the 
pore-to-pore separation and the pore radius were investigated. The results presented show that FIB milling serves as a platform for simple and fast prototyping of nanopore membranes, which are potentially useful in electrochemical and biochemical detection studies.

\section{Results and Discussion}

\subsection{Single and array nanopore preparation by FIB milling}

Figure 1 shows SEM images of the FIB-milled nanopore arrays, which are in a cubic closepacked (CCP) arrangement. The geometric characteristics of the nanopore arrays extracted from these images are listed in Table 1. Nanopores with radii in the range of ca. 30 to $80 \mathrm{~nm}$ were successfully milled in the SiN membranes. Arrays of 9, 16, 25, 100 and 400 pores, featuring the ratio between the pore-to-pore separation, $r_{c}$, and the pore radius, $r_{a}$, in the range of 16 to 32 , were prepared. A large ratio $r_{c} / r_{a}$ has been previously reported to minimise the overlap of diffusion zones formed at adjacent interfaces in electrochemical experiments ${ }^{[5 a, 5 c]}$, as will be discussed in Section 2.2. Although pore shapes in Figure 1 were designed as perfectly round holes, FIB-milling resulted in deviations of the targeted shapes (as clearly visible in Figure 1a). As the membranes are insulating, surface charging led to slight drifts during the milling process. Charging may be omitted by flooding the surface with electrons during bombardment with the positively charged $\mathrm{Ga}^{+}$ions. For the proof-of-principle measurements presented here these minor imperfections should not significantly influence the electrochemical response. However future experiments will focus on improving the disc-shape of the pores using a flood gun during FIB-milling, As shown in Table 1, the precision of the pore sizes is within the range of $10-20 \%$ relative standard deviation. This may be sufficient for a rapid prototyping approach, but improved precision can be obtained with EBL patterning ${ }^{[5 a]}$.

The time for pore formation is dependent on the milling rate, which is greatly influenced by the ion beam parameters such as beam current and dwell time ${ }^{[10 c, 13]}$. The sputter rate mainly depends on ion flux to the sample, the incidence angle of the ion beam, and the probability that 
atoms are ejected from the sample ${ }^{[10 a, 10 c, 19]}$. In this study, the nanopores of design (a) to (f) (Figure 1; Table 1), which employed similar milling parameters, required approximately $0.5 \mathrm{~s}$ for individual pore milling, as measured from a plot of total milling time versus the number of pores (Figure 2). The FIB milling applied here is a serial process in which each pore in the array was being prepared individually ${ }^{[13]}$, resulting in a dependence of the overall milling time on the numbers of pores in the targeted array size, unlike EBL, which enables the parallel formation of pores ${ }^{[5 a]}$. Single and array nanopore electrodes were previously fabricated via FIB milling through a SiN layer over a buried platinum electrode. The time to mill through the $500 \mathrm{~nm} \mathrm{SiN}$ layer with pore radii in the range of $c a .75$ to $200 \mathrm{~nm}$ was typically $40 \mathrm{~s}$ per pore ${ }^{[13]}$. Patterson et al. ${ }^{[20]}$ reported the time to mill through $200 \mathrm{~nm}$ of $\mathrm{SiN}$ was typically within the range 0.1 to $4.0 \mathrm{~s}$, depending on the beam current.

The hydrophobicity of the SiN membrane surface was investigated via water contact angle measurement. The contact angle, $\theta$, is a quantitative measure of the wetting of a solid (the $\operatorname{SiN}$ membrane) by a liquid (deionised water). The measured value was $93.3 \pm 0.5^{\circ}$, confirming a hydrophobic SiN surface. ${ }^{[5 a]}$ An assumption was made that the nanopore walls were analogously hydrophobic, since it was not possible to determine the contact angle inside the nanopores. In addition, due to the high surface-to-volume ratio within the nanopores, it may be difficult to permeate with water, and consequently they behave more hydrophobically in practice compared to the membrane surface ${ }^{[21]}$. Consequently, the organic phase is anticipated to fill the pores in the electrochemical studies presented below.

Membrane porosity is one of the parameters that influence the voltammetric response to ion transfer across the ITIES. It is defined by the cross-sectional area of the pores relative to the whole membrane area. The porosity of the $\mathrm{SiN}$ membrane was calculated from the total area of the membrane surface (in this case $0.25 \mathrm{~mm}^{2}$ ) and the total cross-sectional area of the pores, as described by ${ }^{[8 c]}$ : 
Porosity $=\frac{\pi r_{a}^{2} N_{p}}{A_{\text {total }}} \times 100 \%$

(1)

where $N_{p}$ is the number of pores in the membrane.

The porosity of each membrane studied is shown in Table 1. The values ranged between $0.00048 \%$ and $0.077 \%$, demonstrating the minute fraction of the membrane surface occupied by nanopores. In comparison, a $\gamma$-alumina ultrafiltration membrane previously used for voltammetric experiments exhibited porosity in the range of $13 \%$ to $30 \%{ }^{[8 c]}$.

\subsection{Electrochemical characterisation}

\subsubsection{TPrA ${ }^{+}$transfer across the nanopore membrane-modified ITIES}

The SiN-based single and array nanopore membranes were electrochemically characterised using ion-transfer CV with $\operatorname{TPrA}^{+}$as the model analyte. CV of the background electrolyte solutions was recorded prior to the addition of the analyte ions. Typically, the potential window ranged from 0 to $1 \mathrm{~V}$. In this study, analyte concentrations were varied between 0.1 and $2.0 \mathrm{mM}$ in the aqueous phase solution of $0.01 \mathrm{M} \mathrm{LiCl}$. CVs of five different concentrations of TPrACI were recorded to obtain a calibration curve correlating ion transfer currents with ion concentrations in the aqueous phase. Background-subtracted voltammograms were attained by subtracting the background CV from the CV obtained in the presence of TPrA ${ }^{+}$.

The shape of the voltammetric responses and the magnitude of the currents were dependent on several important parameters, which include: (i) the number of nanopores (equivalent to the number of nanointerfaces), (ii) the pore-to-pore separation, $r_{c}$, (iii) the pore radius, (iv) the recess depth of the liquid | liquid interface position within the pore , $l$, which primarily depends on the hydrophobicity or wetting properties of the membrane material, $(v)$ the diffusion field, whether spherical or linear diffusion occurs at the nanolTIES, and (vi) the magnitude of the diffusion zone extension, $\delta^{[5 a, 22]}$. 
Figure 3 shows voltammograms for the transfer of $\operatorname{TPrA}^{+}$ion from aqueous to organic phase, then back to aqueous phase. CVs were obtained with $2.0 \mathrm{mM}$ and $0.1 \mathrm{mM}$ TPrACl in the aqueous phase, for Figure 3 (a) and (b), respectively. Figure 3 (c) and (d) show the forward scan background-subtracted curves for all concentrations studied. Figure 3 (a) and (c) show results obtained with a single nanoITIES of radius ca. $62 \mathrm{~nm}$, while Figure 3 (b) and (d) show results obtained with an array nanolTIES comprised of $10 \times 10$ interfaces of $c a .47 \mathrm{~nm}$ radius for the individual pores.

Voltammograms of the nanopore membrane-modified ITIES for the forward scan TPrA $^{+}$ion transfer from the aqueous to the organic phase) showed that the current rose steadily with applied potential up to the switching potential. This result is in agreement with previous reports ${ }^{[5 c]}$, where no steady-state (or limiting) current plateau was reached in the diffusion-limited region. The nanolTIES based on design (a) (Figure 3 (a) and (c)) clearly shows this behaviour. The nanolTIES based on design (e) (Figure 3 (b) and (d)), demonstrated an initial diffusionlimited current plateau, but the current then increased gradually with applied potential up to the switching potential. This phenomenon might be explained by a combination of the influence of background electrolyte ion transfer at higher potential ${ }^{[5 c]}$ and reversible expansion of the interface during the ion transfer process ${ }^{[23]}$. Dale and Unwin ${ }^{[23]}$ demonstrated that the polarised liquid | liquid micro-interface was neither flat nor static during voltammetric experiments, as imaged by confocal laser scanning microscopy. The interface underwent significant potentialinduced movement. This increase in interface area may contribute to the observed current response not achieving a limiting plateau, but instead continuing to increase in magnitude. Additionally, the lack of a true limiting current plateau may be due to artefacts introduced by the background-subtraction procedure, although all CVs in Figure 3 show this non-steady-state behaviour. It is important to note that the sloping current in the diffusion-limited region did not occur throughout the voltammogram: it is only observable when the ions start to transfer, as reported previously ${ }^{[5 c]}$. In addition, the nanoITIES based on design (a) (Figure 1 (a)), which is a 
single nanoITIES, exhibited an analyte ion transfer wave that was indistinguishable from the rising current response, in particular, at lower analyte concentration. It is also notable in Figure $3(a$ and $b)$ that the capacitance of the system is significant. Previous studies have reported similar charging effects for membrane designs prepared via EBL. ${ }^{[5 a, d]}$ In such cases, the SiN membrane can be considered as an insulator sandwiched between two conductors (the electrolyte solutions), establishing an electrical capacitor, which contributes a capacitance additional to that of the ITIES ${ }^{[5 d]}$ which is reflected in the recorded CVs.

As no true limiting current plateau is achieved in the diffusion-limited region, the experimentally limiting current was determined at a potential ca. $200 \mathrm{mV}$ positive of the foot of the ion transfer wave, ${ }^{[5 c, 5 d]}$ which was at ca. $0.45 \mathrm{~V}-0.50 \mathrm{~V}$ (Figure 3). It was observed that the ion transfer wave foot progressively shifted to a more negative applied potential with increasing analyte concentration.

In the case of the nanolTIES array (Figure 3 (b) and (d)), the observed forward scan behaviour suggests that there is no diffusion zone overlap as this would introduce linear diffusion to the interfaces, and produce peak-shaped voltammograms. Since the voltammetric behaviour is neither steady-state nor peak-shaped, it is best approximated as a 'sloping steadystate' current behaviour, with establishment of radial (or spherical) diffusion to the interfaces. A recent simulation study demonstrated when radial diffusion to electrodes at the edges of the array is dominant, steady-state characteristics are achieved at nanoelectrode arrays, despite the presence of overlapping diffusion zones at the nanoelectrodes within the array ${ }^{[22 c]}$. The simulation results were supported by experimental data, which are both in good agreement with the results presented here, as well as other studies ${ }^{[13,22 c]}$. The underlying principle of the observed behaviour at nanoelectrode arrays of a few micrometres in size, may be explained that such an array of nanoelectrodes can be treated as a single microelectrode of the same

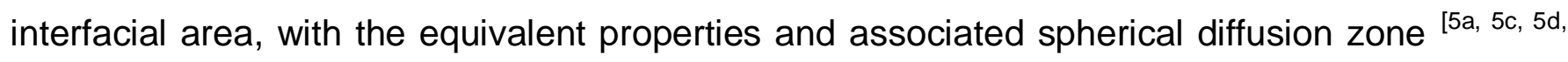
22c]. 


\subsubsection{Influence of the concentration on the limiting current}

The calibration curves correlating the measured current with the concentration of aqueous phase $\operatorname{TPrA}^{+}$are shown in Figure 4 , for single and array $(10 \times 10)$ nanolTIES. Theoretically, for the transfer of ions from the aqueous phase to the organic phase, the liquid | liquid interface can be considered as an inlaid or recessed disk electrode. This geometry depends on whether the nanopores are filled with the organic phase or the aqueous phase. An inlaid nanolTIES is observed when the pore is fully (100\%) filled with the organic phase, such that the interface is positioned at the pore orifice and the interface formed is planar. A recessed nanolTIES is observed when the interface level is lower than the pore orifice.

The current at a single interface can be calculated according to one of the following equations: the inlaid disc current model (given by the Saito, equation 2) or the recessed disc current model (equation 3) ${ }^{[24]}$ :

$I=4|z| F D C r_{a}$

$I=4 \pi|z| F D C r_{a} /\left(4 l+\pi r_{a}\right)$

where $I$ is the current, $F$ is the Faraday constant, and $z, D$ and $C$ are the charge, the diffusion coefficient and the bulk concentration of transferring ions, respectively. $r_{a}$ and $l$ have their usual meanings. To obtain the total current for an array, the current calculated for one nanointerface is multiplied by the number of pores, $N_{p}$. Equation (2) varies from equation (3) by the factor $\left(4 l / \pi r_{a}\right)+1$. When $l$ is zero, equation (3) is analogous to equation (2) ${ }^{[5 c]}$.

In the present study, the interface formed was expected to be inlaid (organic phase solution filled $100 \%$ of the pores) ${ }^{[5 c]}$, thus the theoretical current was estimated by employing equation (2). As expected, a linear relationship exists between the experimental current and $\operatorname{TPrA}^{+}$ concentration for both sets of data in Figure 4. The single nanolTIES (Figure 4 (a)) showed 
good agreement of the experimental current and the calculated current based on the inlaid disc model. This observation supports the hypothesis that the single liquid | liquid interface created was co-planar with the aqueous side of the silicon nitride membrane and consistent with organic phase filling the pores due to the hydrophobicity of the SiN surface and pore walls.

The $10 \times 10$ nanoITIES array (Figure 4 (b)) resulted in measured currents that were approximately $50 \%$ of the theoretical currents calculated using the inlaid disc model modified to include the number of nanointerfaces. This is again in agreement with previous studies ${ }^{[5 c, 5 d, 22 c]}$. This lower current is attributed to the overlap of diffusion zones at adjacent nanointerfaces within the array, so that there is not independent diffusion to each individual nanointerface. The extent of this overlap depends on the relative separation of nanointerfaces (the ratio $r_{c} / r_{a}$ ) ${ }^{[5 a, 5 c]}$. Although the reduced current values reported here could also be associated with recessed rather than inlaid interfaces, the fact that the experimental and theoretical current for a single nanoITIES are in agreement suggests that the nanointerfaces formed in the array format are also inlaid. Previous studies ${ }^{[5 a, 5]}$ have also demonstrated that the interfaces are indeed inlaid and thus implicating diffusion zone overlap as the primary reason for the lower currents.

\subsubsection{Influence of nanopore array geometry}

The influence of nanopore array geometries on the electrochemical behaviour of the nanoITIES was investigated in terms of the number of pores, the average pore-to-pore separation and the average pore radius in the membranes used to form the nanolTIES. The study on the effect of increasing pore numbers $N_{p}$ employed values of 1, 100 and 400 (designs (a), (e) and (f) in Table 1). However, in this case, the pore radius and the $r_{c} / r_{a}$ ratio were not constant as a result of the milling process. The pore radii varied in the range of 39 to $62 \mathrm{~nm}$, with a slight variation in the ratio $r_{c} / r_{a}$ from 20 to 22 . The $\mathrm{CV}$ responses (background-subtracted forward scan only) for the three nanopore array designs for the transfer of $0.1 \mathrm{mM} \mathrm{TPrA}^{+}$across the nanoITIES array are presented in Figure 5. For all designs investigated, the shape of the voltammograms is as 
discussed in the preceding section. As expected, the current increased as $N_{p}$ increased, although not in a linear fashion ${ }^{[5 a]}$. As the number of pores in the array increased, the average current per nanopore decreased ${ }^{[5 a]}$. This is a characteristic of overlapped diffusion zones in an array. Also, it was seen that when $N_{p}$ increased, the $E_{1 / 2}$ value shifted to lower potentials, due to the lower resistance of the array.

Figure 6 summarises the effect of varying the $r_{c} / r_{a}$ ratio on the electrochemical ion transfer signal (here reported as experimental current densities, $j_{\text {exp }}$ ), measured at $10 \times 10$ nanolTIES arrays with radii of ca. $30 \mathrm{~nm}$. For this analysis, design (g) and design (h) were employed, which gave $r_{c} / r_{a}$ ratios of 32 and 17 , respectively. The current density is the ratio of the current, $I$, and the total geometric interfacial area, $A$, which can be obtained from equation (2), in the case of a radial diffusion field:

$j=4|z| F D C / \pi r_{a}$

The total current density for the array is obtained by multiplying the current calculated for one interface by $N_{p}$.

The nanoITIES membrane with the higher ratio $r_{c} / r_{a}$ of 32 (design (g) Table 1) exhibited higher current densities compared to design (h) with $r_{c} / r_{a}$ of 17 (Figure 6). This is consistent with the individual nanointerfaces exhibiting less diffusion zone overlap as they are further displaced from their neighbours. The experimental current density was approximately $5 \%$ and $30 \%$ lower than the calculated current densities for nanoITIES arrays based on design $(\mathrm{g})$ and (h), respectively. The $5 \%$ difference is well within experimental error. Godino et al. ${ }^{[22 c]}$ have demonstrated, via simulations and experiments, that substantial diffusion zone overlap at neighbouring nanoelectrodes occurs, on the timescale of a potential sweep experiment, even for the ratio $r_{c} / r_{a}$ of 60 , which is larger than the commonly used approximation $\left(r_{c} / r_{a}\right.$ of 20) formulated by Fletcher and Horne ${ }^{[22 b, 25]}$ for microelectrode arrays. This latter value is also the 
approximate ratio applied in the present investigation (designs (b), (c), (d), (e), (f) and (h), Table 1).

Table 2 summarises the current, $I$, and current density, $j$, obtained with each of the six nanolTIES arrays (designs (a), (b), (e), (f), (g) and (h), Table 1) for transfer of $100 \mu \mathrm{M}$ TPrA ${ }^{+}$ $I_{\text {calc }}$ and $I_{\text {calc,total }}$ are obtained using equation (2), while $I_{\text {exp }}$ is obtained from CV experiments. $j_{\text {calc }}$ was obtained employing equation (4) and $j_{\text {exp }}$ is the experimental current divided by the total geometric area of the interfaces. The individual experimental current and current densities were in the range of 23 to $61 \%$ lower than the calculated values, which is supportive of the possibility of diffusion zone overlap. In the case of design (g), the experimental current was within $60 \%$ of the calculated current (equation (2)), suggesting that the interfaces formed at the nanopore orifices were not flat. In general, it can be seen that as pore size changes, so do the current and the current density, in line with expectations.

\subsubsection{Influence of the tetraalkylammonium species on the transfer process}

Figure 7 shows voltammograms for the transfer of three different tetraalkylammonium cations $\left(\mathrm{TEA}^{+}, \mathrm{TPrA}^{+}\right.$and $\left.\mathrm{TBA}^{+}\right)$(concentration of $\left.500 \mu \mathrm{M}\right)$ across the nanolTIES array utilising a $10 \times$ 10 nanopore array membrane (design (e)). As expected, the cations transferred in the order of their affinity for the organic phase. The more hydrophobic cations, $\mathrm{TBA}^{+}$, transfer at the lowest applied potential $\left(E_{1 / 2}\right.$ of $\left.0.40 \mathrm{~V}\right)$, while the more hydrophilic cations, $\mathrm{TEA}^{+}$, transfer at the highest applied potential $\left(E_{1 / 2}\right.$ of $\left.0.68 \mathrm{~V}\right)$, following the sequence $\mathrm{TBA}^{+}<\mathrm{TPrA}^{+}<\mathrm{TEA}^{+}$. The differences observed between the respective $\mathrm{TAA}^{+}$limiting currents are due to their diffusion coefficients, $D$. The values of the diffusion rate for TEA ${ }^{+}, \operatorname{TPrA}^{+}$and $\mathrm{TBA}^{+}$are $8.4 \times 10^{-6} \mathrm{~cm}^{2} \mathrm{~s}^{-1}$, $7.5 \times 10^{-6} \mathrm{~cm}^{2} \mathrm{~s}^{-1}$ and $6.0 \times 10^{-6} \mathrm{~cm}^{2} \mathrm{~s}^{-1}$, respectively ${ }^{[26]}$. The CVs showed that currents in the limiting-current region rose with the applied potential up to the switching potential, irrespective of the analyte. 


\section{Conclusion}

For the first time, nanopores prepared by FIB milling were used to nanostructure the liquid I liquid interface, thereby forming the nanoITIES. Single and array nanoporous SiN membranes were prepared with radii in the range of 30 to $80 \mathrm{~nm}$, and with pore-to-pore separation ratios of 16 to 32 in SiN films of thickness $50 \mathrm{~nm}$. These membranes were electrochemically characterised via the formation of nanoscale-liquid | liquid interfaces and the study of CVs of $\mathrm{TPrA}^{+}$ion transfer across the interface between water and $\mathrm{DCH}$. While steady-state voltammograms were achieved, the limiting current region demonstrated an increase of current with applied potential up to the switching potential at all the nanolTIES studied. A single nanoITIES gave excellent agreement between the experimental current and the theoretical current for an inlaid disc interface, showing that such FIB-milled nanopores enable the formation of inlaid nanointerfaces that are co-planar with the aqueous side of the membrane. At nanoITIES arrays, experimental currents lower than calculated currents were thus attributed to diffusion zone overlap. Nevertheless, experimental currents were dependent, as expected, on the nanopore size, the pore-to-pore separation and the numbers of pores in the array used to nanostructure the interface. The results show that FIB-milled nanopores can be used for prototyping of the nanoITIES arrays and open up the possibilities for new chemical and biochemical sensing systems.

\section{Experimental Section}

\subsection{Materials and reagents}

The silicon nitride membranes (DuraSiN ${ }^{\mathrm{TM}}$ film) were purchased from Electron Microscopy Sciences, Pennsylvania, USA. The SiN film is supported on a rigid silicon frame $(2.65 \mathrm{~mm} \times$ $2.65 \mathrm{~mm} \times 300 \mu \mathrm{m})$. All membranes used for FIB-milling had a thickness film of $50 \mathrm{~nm}$.

All chemical reagents were obtained from Sigma Aldrich, Australia and were used as received, unless stated otherwise. The supporting electrolytes in the aqueous and organic 


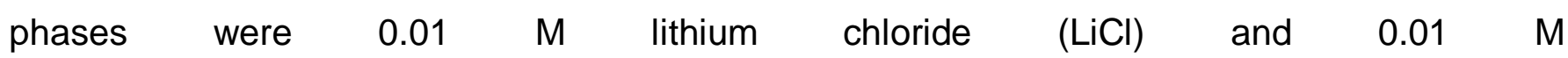
bis(triphenylphosphoranylidene)ammonium tetrakis(4-chlorophenyl)borate (BTPPATPBCI), respectively. Both the aqueous and organic phase solvents were mutually pre-saturated, prior to experiments. The organic electrolyte salt was prepared by metathesis reaction of bis(triphenylphosphoranylidene)ammonium chloride (BTPPACI) and potassium tetrakis(4chlorophenyl)borate (KTPBCI). The organic reference solution was $0.01 \mathrm{M} B T P P A C l$ dissolved in aqueous $0.01 \mathrm{M} \mathrm{LiCl}$. The primary tetraalkylammonium salt used as model analyte in this study was the chloride salt of $\mathrm{TPrA}^{+}$in $0.01 \mathrm{M} \mathrm{LiCl}$ in 1,6-dichlorohexane (DCH)-saturated water. In addition, tetraethylammonium $\left(\mathrm{TEA}^{+}\right)$and tetrabutylammonium $\left(\mathrm{TBA}^{+}\right)$used as analytes were in their chloride and bromide forms, respectively.

\subsection{Nanopore preparation by FIB milling}

The DuraSiN ${ }^{\mathrm{TM}}$ membranes were prepared for milling by securing them to the SEM stubs using copper tape or silver paint to facilitate easy removal. Single and array pores were milled in the $500 \mu \mathrm{m} \times 500 \mu \mathrm{m}$ SiN film window using either a focused ion beam scanning electron microscope (FIBSEM) instrument, Zeiss Neon 40EsB (Carl Zeiss Nano Technology Systems, Oberkochen, Germany), at the John de Laeter Centre, Curtin University, Australia, or a DualBeam Helios Nanolab 600 FIB/SEM (FEI Company, Eindhoven, NL) at the FIB Centre, Institute of Analytical and Bioanalytical Chemistry, University of Ulm, Germany. All pores were milled using gallium ions with beam acceleration voltage of $30.0 \mathrm{kV}$. The milling current was either $50 \mathrm{pA}$ (on the Zeiss Neon 40EsB), which corresponds to an ion beam probe size of 25.0 $\mathrm{nm}$, or $10 \mathrm{pA}$ (on the DualBeam Helios Nanolab 600), resulting in an ion beam probe size of $12.8 \mathrm{~nm}$.

The milled pores were imaged and characterised by SEM with an acceleration voltage of 5 $\mathrm{kV}$ or $3 \mathrm{kV}$, utilising the Zeiss Neon 40EsB or DualBeam Helios Nanolab 600 instruments, 
respectively. Nanopore geometry measurements from the SEM images were conducted with ImageJ software (National Institutes of Health, Maryland, USA).

\subsection{Contact angle measurement}

Contact angle measurements were conducted using a Contact Angle Meter, CAM 101 (KSV Instrument Ltd., Helsinki, Finland). The contact angle (water/air/membrane) between the dispensed drop (ca. $1 \mu \mathrm{L}$ ) and the substrate surface was measured directly after the contact was created.

\subsection{Preparation of nanopore-supported ITIES}

The single and array nanolTIES were formed at a water|DCH interface. The membranes were sealed onto the lower orifice of cylindrical borosilicate glass tubes $\left(d_{\text {inner }}=1.4 \mathrm{~mm}, d_{\text {outer }}=3.0\right.$ $\mathrm{mm}$ ) using silicone rubber sealant (Selleys, Australia and New Zealand) and allowed to cure for 72 hours prior to first usage. The organic phase electrolyte $(10 \mu \mathrm{L})$ and the organic reference solution $(200 \mu \mathrm{L})$ were added into the borosilicate glass tube. This assembly was then immersed in $6 \mathrm{~mL}$ of the aqueous phase solution contained in a $10 \mathrm{~mL}$ glass beaker. A twoelectrode electrochemical cell with two $\mathrm{Ag} \mid \mathrm{AgCl}$ electrodes was employed in this work. The cell set up was placed in a Faraday cage to minimise electrical noise. The electrochemical cell can be summarized as follows:

$\mathrm{Ag}|\mathrm{AgCl}| x \mathrm{mM}$ TAAX $+0.01 \mathrm{M} \mathrm{LiCl}_{\mathrm{W}}|| 0.01 \mathrm{M} \mathrm{BTPPATPBCl} \mathrm{DCH} \mid 0.01 \mathrm{M} \mathrm{BTPPACl}+0.01 \mathrm{M}$

$$
\mathrm{LiCl}_{W}|\mathrm{AgCl}| \mathrm{Ag}
$$

where $x$ is the concentration of tetraalkylammonium salt (TAAX) in the aqueous phase (TEACI, TPrACl and $\mathrm{TBABr}$ ). All potentials are reported here relative to the experimentally-used reference electrodes.

\subsection{Electrochemical procedure}


Following assembly of the electrochemical cell, CV was applied using an Autolab PGSTAT 302N (Metrohm Autolab B. V., Utrecht, The Netherlands) interfaced to a personal computer, running the Nova software package supplied with the instrument. A background voltammogram (i.e. $x=0$ in the above electrochemical cell) was recorded over a wide potential range to establish the limits of the available potential window before injection of the required amount of $\mathrm{TPrA}^{+}$concentrated solution into the aqueous phase. A $5 \mathrm{~s}$ quiet time was employed at the initial applied potential prior to recording each CV in order to stabilize the background charging current. Throughout this study, a voltammetric sweep rate of $5 \mathrm{mV} \mathrm{s}^{-1}$ was employed.

\section{Acknowledgements}

This research was supported in part by the Australian Research Council (DP130102040) and the Malaysian Agricultural Research and Development Institute (MARDI), Malaysia. The authors acknowledge the use of equipment, scientific and technical assistance of the John de Laeter Centre, Curtin University, which has been partially funded by the University, and by the State and Commonwealth Governments. The Focused Ion Beam Centre, University of Ulm, is supported by FEl Company (Eindhoven, Netherlands), the German Science Foundation (INST40/385-F1UG), and the Struktur- und Innovationsfonds Baden-Württemberg. CK acknowledges the financial support from Deutscher Akademischer Austausch Dienst (DAAD) within the project 57060632 . The authors are grateful to the Joint Research Co-operation Scheme of the Australian Technology Network of Universities and the DAAD for provision of a travel grant.

Keywords: nanopore $\bullet$ liquid | liquid interface $\bullet$ focused ion beam $\bullet$ cyclic voltammetry • nanoelectrochemistry

[1] a) H. H. Girault, in Modern Aspects of Electrochemistry, Vol. 25 (Ed.: J. O. M. Bockris), Plenum Press, New York, 1993, pp. 1-62; b) P. Vanysek, Trends Anal. Chem. 1993, 12, 357-363; c) Z. 
Samec, Pure Appl. Chem. 2004, 76, 2147-2180; d) H. H. Girault, in Electroanalytical Chemistry: A Series of Advances, Vol. 23 (Eds.: A. J. Bard, C. G. Zoski), CRC Press, Boca Raton FL, 2010, pp. 1104.

[2] D. W. M. Arrigan, G. Herzog, M. D. Scanlon, J. Strutwolf, in Electroanalytical Chemistry: A Series of Advances, Vol. 25 (Eds.: A. J. Bard, C. G. Zoski), CRC Press, Boca Raton FL, 2013, pp. 105-178.

[3] S. Amemiya, J. Kim, A. Izadyar, B. Kabagambe, M. Shen, R. Ishimatsu, Electrochim. Acta 2013, 110, 836-845.

[4] S. Amemiya, Y. Wang, M. V. Mirkin, in Specialist Periodical Reports in Electrochemistry:

Nanoelectrochemistry, Vol. 12 (Eds.: R. G. Compton, J. D. Wadhawan), RSC Publishing Cambridge, UK, 2014, pp. 1-43.

[5] a) M. D. Scanlon, J. Strutwolf, A. Blake, D. Iacopino, A. J. Quinn, D. W. M. Arrigan, Anal. Chem. 2010, 82, 6115-6123; b) M. D. Scanlon, D. W. M. Arrigan, Electroanal. 2011, 23, 1023-1028; c) M. Rimboud, R. D. Hart, T. Becker, D. W. M. Arrigan, Analyst 2011, 136, 4674-4681; d) M. Sairi, J. Strutwolf, R. A. Mitchell, D. S. Silvester, D. W. M. Arrigan, Electrochim. Acta 2013, 101, 177-185.

[6] a) Y. Shao, M. V. Mirkin, J. Am. Chem. Soc. 1997, 119, 8103-8104; b) P. Sun, Z. Zhang, Z. Gao, Y. Shao, Angew. Chem., Int. Ed. 2002, 41, 3445-3448; c) F. O. Laforge, P. Sun, M. V. Mirkin, J. Am. Chem. Soc. 2006, 128, 15019-15025.

[7] B. Liu, Y. Shao, M. V. Mirkin, Anal. Chem. 2000, 72, 510-519.

[8] a) R. A. W. Dryfe, B. Kralj, Electrochem. Commun. 1999, 1, 128-130; b) B. Kralj, R. A. W. Dryfe, Phys. Chem. Chem. Phys. 2001, 3, 5274-5282; c) M. Platt, R. A. W. Dryfe, E. P. L. Roberts, Langmuir 2003, 19, 8019-8025.

[9] a) H. D. Tong, H. V. Jansen, V. J. Gadgil, C. G. Bostan, E. Berenschot, C. J. M. van Rijn, M. Elwenspoek, Nano Lett. 2004, 4, 283-287; b) L. D. Menard, J. M. Ramsey, Nano Lett. 2011, 11, 512-517; c) S. P. Branagan, N. M. Contento, P. W. Bohn, J. Am. Chem. Soc. 2012, 134, 8617-8624.

[10] a) M. J. Kim, B. McNally, K. Murata, A. Meller, Nanotechnology 2007, 18, 205302/205301205302/205305; b) M. Y. Wu, D. Krapf, M. Zandbergen, H. Zandbergen, P. E. Batson, Appl. Phys. Lett. 2005, 87, 113106/113101-113106/113103; c) D. Krapf, M. Y. Wu, R. M. M. Smeets, H. W. Zandbergen, C. Dekker, S. G. Lemay, Nano Lett. 2006, 6, 105-109; d) C. Ho, R. Qiao, J. B. Heng, A. Chatterjee, R. J. Timp, N. R. Aluru, G. Timp, K. Hess, Proc. Natl. Acad. Sci. U.S.A. 2005, 102, 1044510450.

[11] A. Han, M. Creus, G. Schurmann, V. Linder, T. R. Ward, N. F. de Rooij, U. Staufer, Anal. Chem. 2008, 80, 4651-4658.

[12] a) P. S. Spinney, D. G. Howitt, R. L. Smith, S. D. Collins, Nanotechnology 2010, 21, 375301/375301-375301/375307; b) M. Yemini, B. Hadad, Y. Liebes, A. Goldner, N. Ashkenasy, Nanotechnology 2009, 20, 245302/245301-245302/245306.

[13] Y. H. Lanyon, G. D. Marzi, Y. E. Watson, A. J. Quinn, J. P. Gleeson, G. Redmond, D. W. M. Arrigan, Anal. Chem. 2007, 79, 3048-3055.

[14] M. Lillo, D. Losic, Mater. Lett. 2009, 63, 457-460.

[15] C. Dekker, Nat. Nanotechnol. 2007, 2, 209-215.

[16] J. Li, D. Stein, C. McMullan, D. Branton, M. J. Aziz, J. A. Golovchenko, Nature 2001, 412, 166-169.

[17] J. J. Kasianowicz, E. Brandin, D. Branton, D. W. Deamer, Proc. Natl. Acad. Sci. U.S.A. 1996, 93, 13770-13773.

[18] H. Chang, F. Kosari, G. Andreadakis, M. A. Alam, G. Vasmatzis, R. Bashir, Nano Lett. 2004, 4, 1551-1556.

[19] A. J. Storm, J. H. Chen, X. S. Ling, H. W. Zandbergen, C. Dekker, Nat. Mater. 2003, 2, 537-540.

[20] N. Patterson, D. P. Adams, V. C. Hodges, M. J. Vasile, J. R. Michael, P. G. Kotula, Nanotechnology 2008, 19, 235304/235301-235304/235309.

[21] D. Bratko, C. D. Daub, K. Leung, A. Luzar, J. Am. Chem. Soc. 2007, 129, 2504-2510. 
[22] a) D. W. M. Arrigan, Analyst 2004, 129, 1157-1165; b) T. J. Davies, R. G. Compton, J. Electroanal. Chem. 2005, 585, 63-82; c) N. Godino, X. Borrise, F. X. Munoz, F. J. del Campo, R. G. Compton, J. Phys. Chem. C 2009, 113, 11119-11125.

[23] S. E. C. Dale, P. R. Unwin, Electrochem. Commun. 2008, 10, 723-726.

[24] a) Y. Saito, Rev. Polarog. 1968, 15, 177-187; b) A. M. Bond, D. Luscombe, K. B. Oldham, C. G. Zoski, J. Electroanal. Chem. 1988, 249, 1-14.

[25] S. Fletcher, M. D. Horne, Electrochem. Commun. 1999, 1, 502-512.

[26] J. Langmaier, K. Stejskalova, Z. Samec, J. Electroanal. Chem. 2001, 496, 143-147.

[27] J. C. Miller, J. N. Miller, Statistics for Analytical Chemistry, 2nd ed., Ellis Horwood Limited, England, 1988. 
Table 1. Characteristics of the eight different nanopore array membranes prepared by FIB milling

\begin{tabular}{lllllll}
\hline Design & Arrays & $\begin{array}{l}\text { Number } \\
\text { of pores } \\
\text { in the } \\
\text { array, } N_{p}\end{array}$ & $\begin{array}{l}\text { Pore } \\
\text { radius, } \\
r_{a} \\
(\mathrm{~nm})^{[a]} \\
( \pm \mathrm{x})\end{array}$ & $\begin{array}{l}\text { Pore-to- } \\
\text { pore } \\
\text { separation, } \\
r_{c}(\mathrm{~nm})^{[\mathrm{b}]} \\
( \pm \mathrm{x})\end{array}$ & $\begin{array}{l}r_{c} / r_{a}^{[\mathrm{c}]} \\
( \pm \mathrm{x})\end{array}$ & $\begin{array}{l}\text { Porosity, \% } \\
( \pm \mathrm{x})\end{array}$ \\
& & & $62 \pm 3$ & - & - & \\
\hline $\mathrm{a}$ & $1 \times 1$ & 1 & $62]$ & & \\
$\mathrm{b}$ & $3 \times 3$ & 9 & $80 \pm 15$ & $1312 \pm 27$ & $16 \pm 3$ & $7.24( \pm 0.47) \times 10^{-6}$ \\
$\mathrm{c}$ & $4 \times 4$ & 16 & $66 \pm 9$ & $1197 \pm 41$ & $18 \pm 3$ & $8.76( \pm 2.39) \times 10^{-5}$ \\
$\mathrm{~d}$ & $5 \times 5$ & 25 & $66 \pm 8$ & $1162 \pm 26$ & $18 \pm 2$ & $1.37( \pm 0.33) \times 10^{-4}$ \\
$\mathrm{e}$ & $10 \times 10$ & 100 & $47 \pm 6$ & $1019 \pm 65$ & $22 \pm 3$ & $2.78( \pm 0.71) \times 10^{-4}$ \\
$\mathrm{f}$ & $20 \times 20$ & 400 & $39 \pm 6$ & $778 \pm 20$ & $20 \pm 3$ & $7.65( \pm 2.35) \times 10^{-4}$ \\
$\mathrm{~g}$ & $10 \times 10$ & 100 & $31 \pm 3$ & $996 \pm 4$ & $32 \pm 3$ & $1.21( \pm 0.23) \times 10^{-4}$ \\
$\mathrm{~h}$ & $10 \times 10$ & 100 & $30 \pm 1$ & $497 \pm 2$ & $17 \pm 1$ & $1.13( \pm 0.08) \times 10^{-4}$ \\
\hline
\end{tabular}

[x] Standard deviation

[a] Pore radius are average values over 5 pores with 3 measurements in each pore, with the exception of design (a) where 3 measurements were taken of the pore

[b] Pore-to-pore separation are average values over 5 measurements

[c] The standard deviation of $r_{c} / r_{a}$ ratio and porosity were calculated using the method of propagation of random errors ${ }^{[27]}$ 
Table 2. Summary of the current, $I$ and current density, $j$ obtained by $C V$ of $100 \mu \mathrm{M} \operatorname{TPrA}^{+}$of nanolTIES formed at nanoporous membrane

\begin{tabular}{llllll}
\hline Design & $\begin{array}{l}I_{\text {calc }} \text { per pore }(\mathrm{nA})^{[\mathrm{a}]} \\
( \pm \mathrm{x} \%)\end{array}$ & $\begin{array}{l}I_{\text {calc,total }}(\mathrm{nA})^{[\mathrm{b}]} \\
( \pm \mathrm{x} \%)\end{array}$ & $\begin{array}{l}I_{\text {exp }}(\mathrm{nA})^{[\mathrm{c}]} \\
( \pm \mathrm{x} \%)\end{array}$ & $\begin{array}{l}j_{\text {calc }}\left(\mathrm{mA} \mathrm{cm}^{-2}\right)^{[\mathrm{d}]} \\
( \pm \mathrm{x} \%)\end{array}$ & $\begin{array}{l}j_{\text {exp }}\left(\mathrm{mA} \mathrm{cm}^{-2}\right)^{[\mathrm{e}]} \\
( \pm \mathrm{x} \%)\end{array}$ \\
\hline $\mathrm{a}$ & $1.79 \times 10^{-3}( \pm 5)$ & $1.79 \times 10^{-3}( \pm 5)$ & $1.22 \times 10^{-3}( \pm 7)$ & $15( \pm 11)$ & $10( \pm 12)$ \\
$\mathrm{b}$ & $2.32 \times 10^{-3}( \pm 19)$ & $2.08 \times 10^{-2}( \pm 19)$ & $1.61 \times 10^{-2}( \pm 2)$ & $12( \pm 42)$ & $9( \pm 38)$ \\
$\mathrm{e}$ & $1.36 \times 10^{-3}( \pm 13)$ & $1.36 \times 10^{-1}( \pm 13)$ & $9.22 \times 10^{-2}( \pm 32)$ & $20( \pm 29)$ & $13( \pm 41)$ \\
$\mathrm{f}$ & $1.13 \times 10^{-3}( \pm 15)$ & $4.52 \times 10^{-1}( \pm 15)$ & $1.75 \times 10^{-1}( \pm 23)$ & $24( \pm 34)$ & $9( \pm 39)$ \\
$\mathrm{g}$ & $8.97 \times 10^{-4}( \pm 10)$ & $8.97 \times 10^{-2}( \pm 10)$ & $1.43 \times 10^{-1}( \pm 11)$ & $30( \pm 22)$ & $47( \pm 22)$ \\
$\mathrm{h}$ & $8.68 \times 10^{-4}( \pm 3)$ & $8.68 \times 10^{-2}( \pm 3)$ & $5.08 \times 10^{-2}( \pm 6)$ & $31( \pm 7)$ & $18( \pm 9)$ \\
\hline
\end{tabular}

[a] $I_{\text {calc }}$ is the calculated current (using equation (2)) for a given pore radius assuming an inlaid liquid | liquid interface

[b] $I_{\text {calc,total }}$ is obtained by multiplying the current per pore and the number of pores in the array

[c] $I_{\text {exp }}$ is the measured current from cyclic voltammetry experiment

[d] $j_{\text {calc }}$ is the calculated current density (using equation (4)) employing a diffusion coefficient of the analyte of $7.5 \times 10-10 \mathrm{~m}^{2} \mathrm{~s}^{-1}$ $[\mathrm{e}] j_{\text {exp }}$ is the experimental current density

$[x] \%$ relative standard deviation (\% r.s.d.). All standard deviations of $I$ and $j$ were calculated using the method of propagation of random errors ${ }^{[27]}$ 

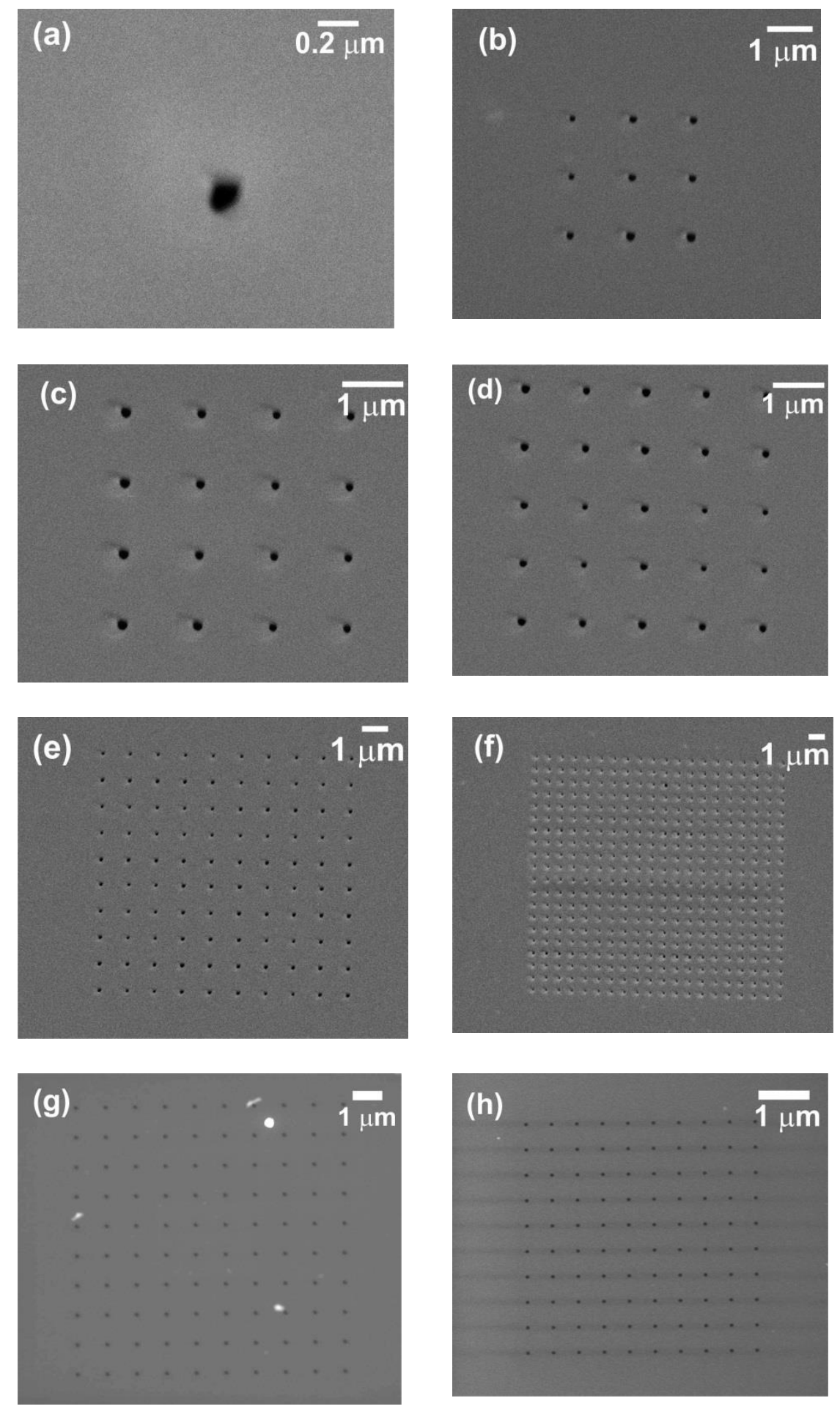

Figure 1. SEM images of the FIB-milled single and array nanopore membranes with $50 \mathrm{~nm}$ film thickness. (a) $1 \times 1$ (b) $3 \times 3$ (c) $4 \times 4$ (d) $5 \times 5$ (e) $10 \times 10$ and (f) $20 \times 20$ nanopore arrays prepared via Zeiss Neon $40 \mathrm{EsB} .(\mathrm{g})$ and $(\mathrm{h})$ represent $10 \times 10$ nanopore arrays prepared via DualBeam Helios Nanolab 600 with pore-to-pore 
separation, $r_{c}$ ca. $1000 \mathrm{~nm}$ and $500 \mathrm{~nm}$, respectively. All the nanopore membranes featured a cubic close-packed (CCP) arrangement. 


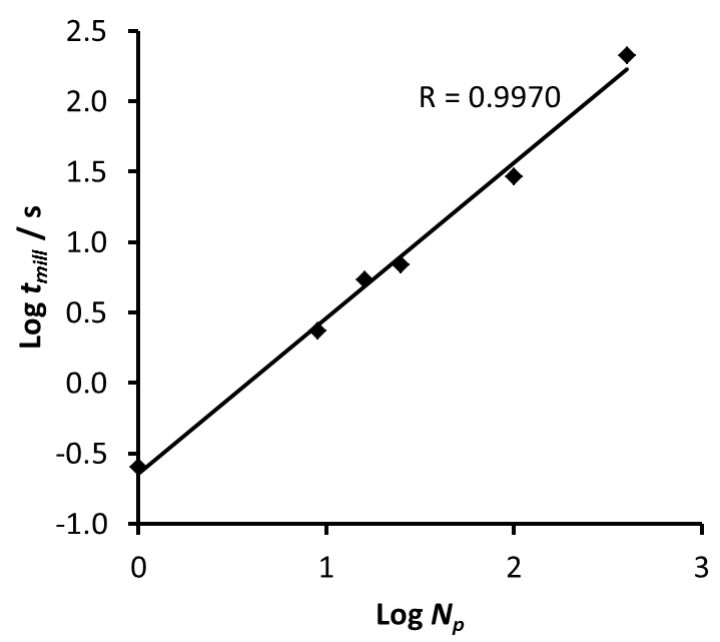

Figure 2. Plot of the dependence of total milling time versus number of pores milled in $50 \mathrm{~nm}$ thick $\mathrm{SiN}$ film with $30 \mathrm{kV}$ acceleration voltage and $50 \mathrm{pA}$ milling current. Both axes are in log scales. 


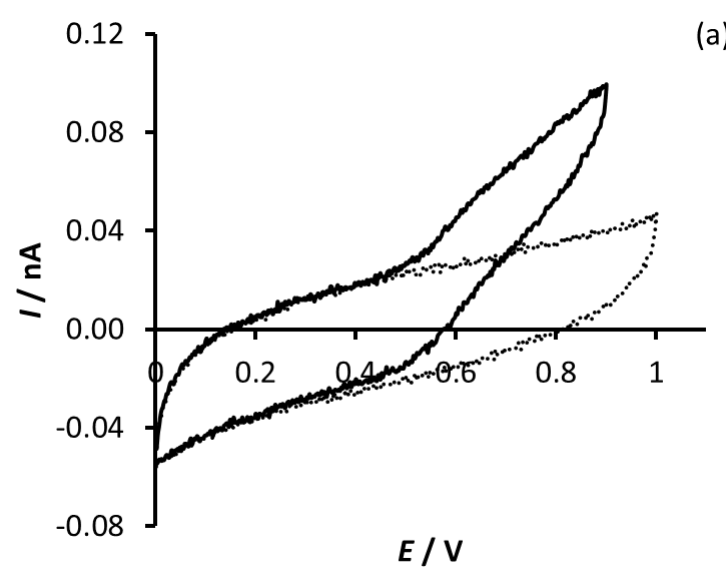

(a)
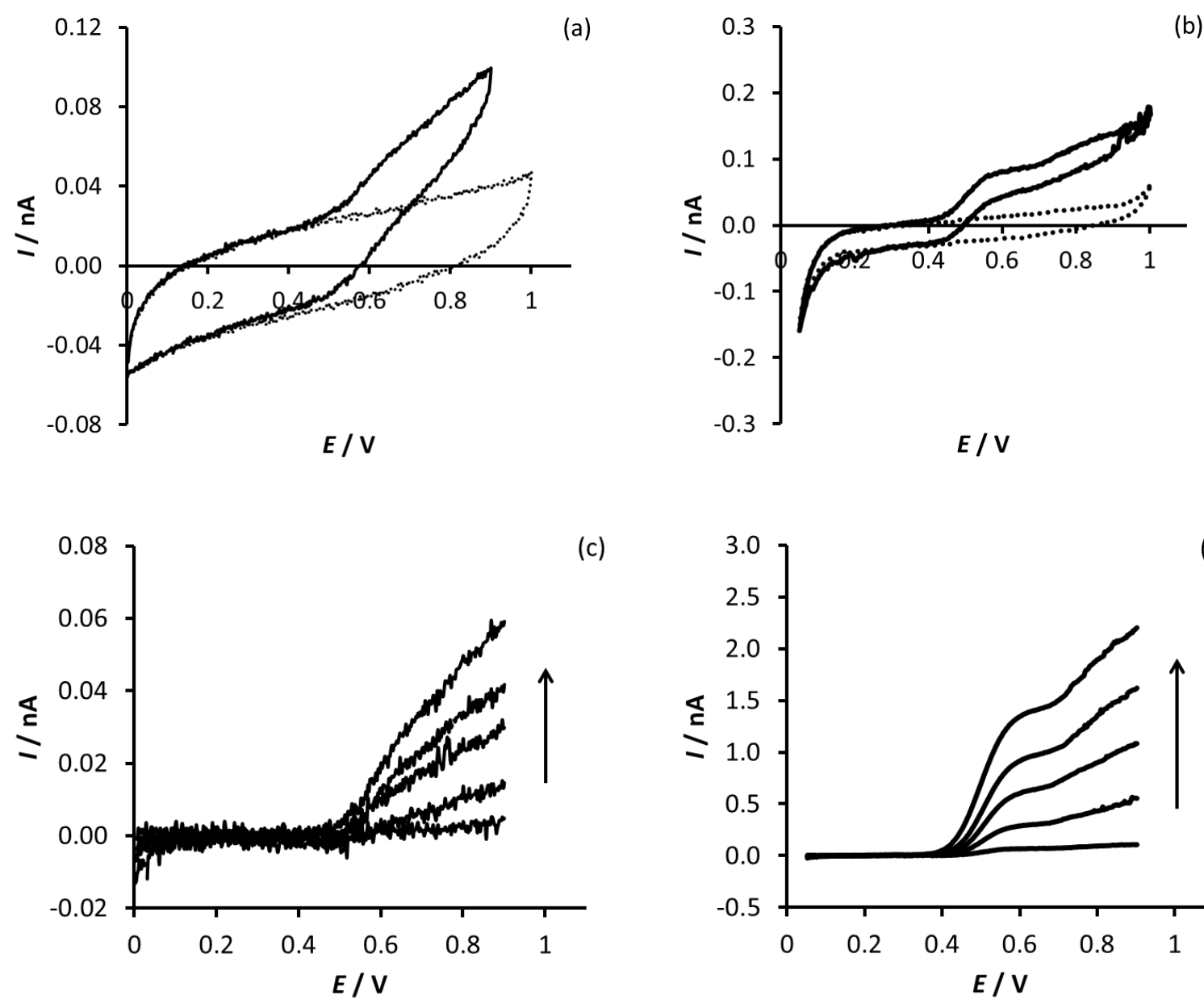

(c)

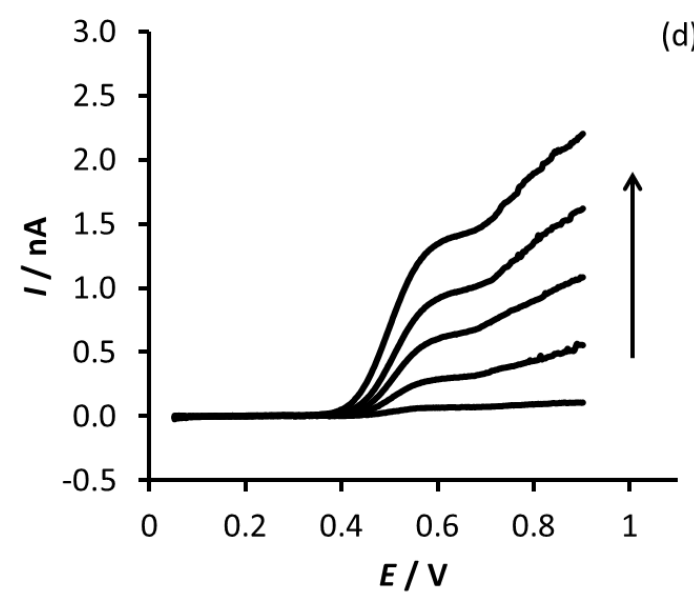

Figure 3. CVs of (a) $2.0 \mathrm{mM}$, and (b) $0.1 \mathrm{mM}$ tetrapropylammonium cation $\left(\operatorname{TPrA}^{+}\right)$ transfer across water|1,6-dichlorohexane $(\mathrm{DCH})$ interface formed within a nanoporous silicon nitride membrane, at $5 \mathrm{mV} \mathrm{s}^{-1}$ sweep rate. The dotted and solid lines represent blank and analyte voltammograms, respectively. (c), (d) Backgroundsubtracted voltammograms (forward scan only) of $0.1,0.5,1.0,1.5$ and $2.0 \mathrm{mM}$ $\mathrm{TPrA}^{+}$transfer across the nanoITIES with arrows indicate increasing analyte concentrations. Figure (a) and (c) represent single nanopore membrane, $r_{a}$ of $62 \pm 3$ $\mathrm{nm}$ (design (a) in Table 1), while figure (b) and (d) represent array $(10 \times 10)$ nanopore membrane, $r_{a}$ of $47 \pm 6 \mathrm{~nm}$ (design (e) in Table 1). 

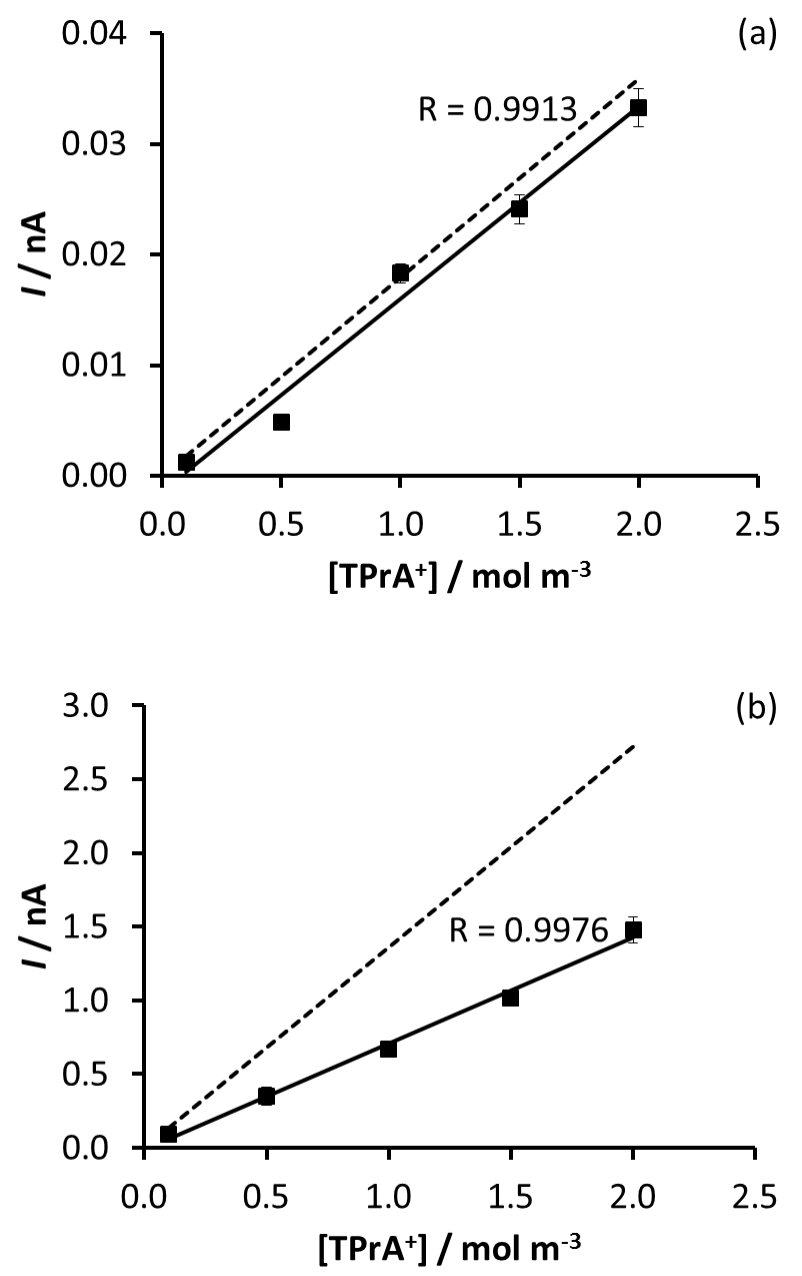

Figure 4. The calibration curve plots of the experimental currents (forward scans) against $\operatorname{TPrA}^{+}$concentrations, represented by squares. The dashed lines represent the theoretical inlaid disc model current based on equation (2) while the solid lines are the best linear fit to the experimental data. (a) and (b) represent single $\left(r_{a}\right.$ of $62 \pm$ $3 \mathrm{~nm}$, design (a)) and array $(10 \times 10)\left(r_{a}\right.$ of $47 \pm 6 \mathrm{~nm}$, design (e)) nanopore membranes, respectively. 


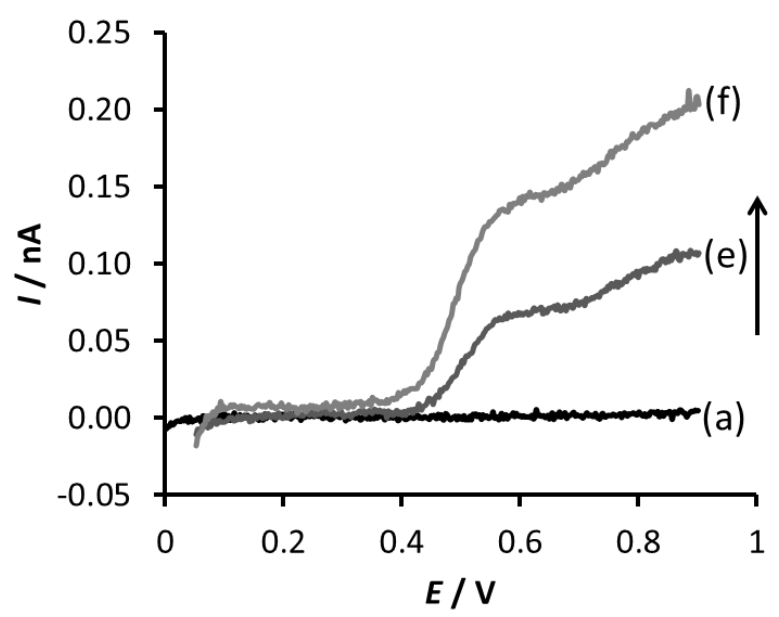

Figure 5. Background-subtracted CV (forward sweep only) of $100 \mu \mathrm{M} \operatorname{TPrA}^{+}$ion transfer at single and array nanoITIES created using nanopore design (a: $r_{a}$ of $62 \pm 3$ $\mathrm{nm}$ ), (e: $r_{a}$ of $\left.47 \pm 6 \mathrm{~nm}\right)$ and (f: $r_{a}$ of $39 \pm 6 \mathrm{~nm}$ ) with increasing number of pores in the array, $N_{p}$ of 1,100 and 400 pores, accordingly. Arrow indicates an increase in $N_{p}$. Scan rate used was $5 \mathrm{mV} \mathrm{s}^{-1}$. 


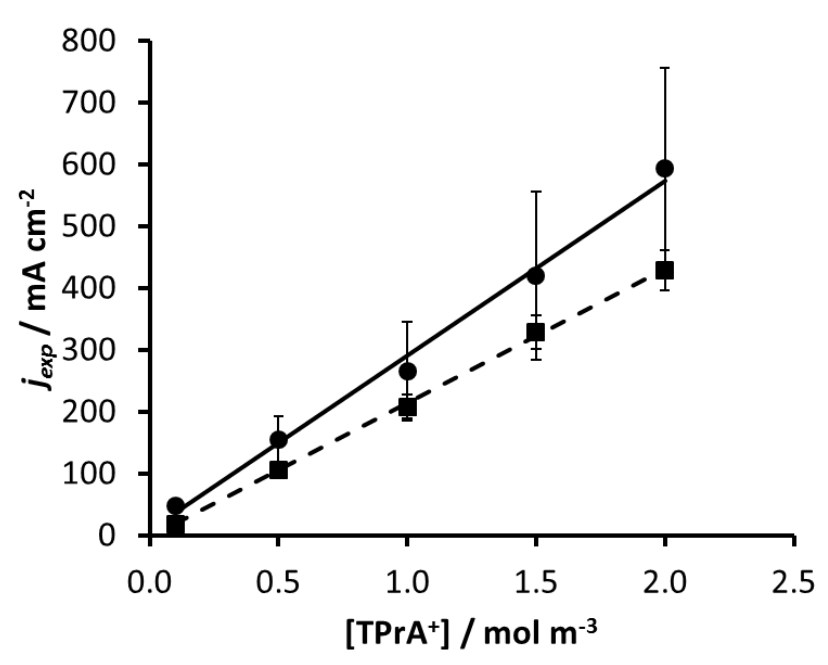

Figure 6. Comparison of the current densities, $j_{\exp }\left(\mathrm{mA} \mathrm{cm}^{-2}\right)$ featuring the ratio of $r_{c} / r_{a}$ of 32 (design (g), solid line) or 17 (design (h), dashed line). $\operatorname{TPrA}^{+}$concentration $=0.1$ to $2.0 \mathrm{mM} ; 10 \times 10$ nanoITIES arrays of $r_{a}=30 \mathrm{~nm}$; scan rate $=5 \mathrm{mV} \mathrm{s}^{-1}$. 


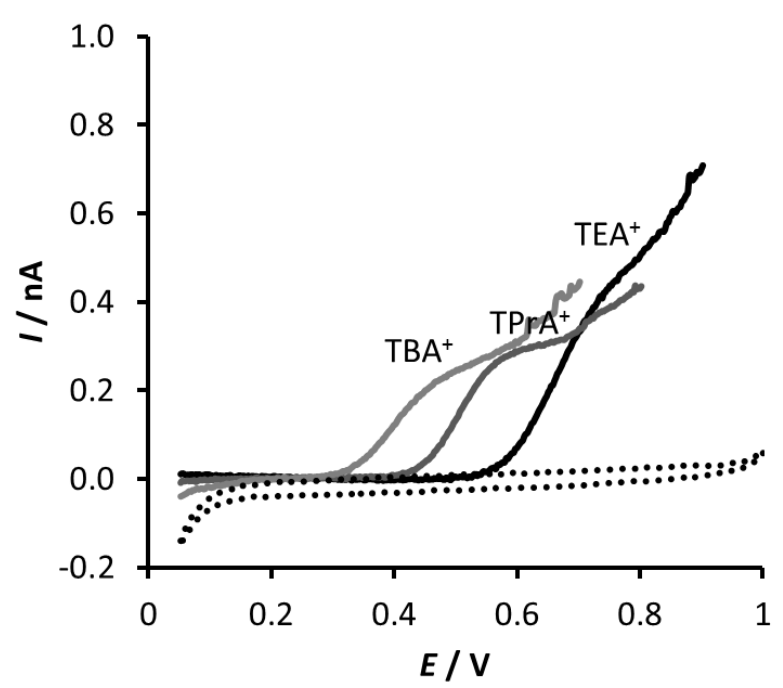

Figure 7. Background subtracted CV (forward scan only) recorded for $500 \mu \mathrm{M}$ of $\mathrm{TEA}^{+}, \mathrm{TPrA}^{+}$and $\mathrm{TBA}^{+}$at water | 1,6-dichlorohexane interface obtained with nanopore array design (e) $\left(r_{a}\right.$ of $\left.47 \pm 6 \mathrm{~nm}\right)$. Dotted line represents the blank voltammogram. 
